\title{
Melanoma in pregnancy: certainties unborn
}

\author{
Enrico Zelin ${ }^{1}$, Claudio Conforti1 ${ }^{(i D}$, Roberta Giuffrida*,2(iD), Teresa Deinlein ${ }^{3}$ Nicola di \\ Meo $^{1}$ (iD) \& Iris Zalaudek ${ }^{1}$ (iD \\ ${ }^{1}$ Dermatology \& Venereology Department, Dermatology Clinic, Maggiore Hospital, University of Trieste, Trieste, Italy \\ ${ }^{2}$ Department of Clinical \& Experimental Medicine, Dermatology, University of Messina, Messina, Italy \\ ${ }^{3}$ Department of Dermatology, Medical University of Graz, Graz, Austria \\ *Author for correspondence: roberta_giuffrida@hotmail.it
}

\section{Practice points}

- To date, pregnancy-associated melanoma (PAM) has been reported to have $17-56 \%$ higher mortality than melanoma in nonpregnant women.

- The role of sexual hormones, cytokines and circulating factors, the immune status changes and the increased lymphangiogenesis during pregnancy are biological mechanisms that could explain the worse outcome of PAM; however, the poorer prognosis could be simply due to diagnostic and therapeutic delay.

- If melanoma is suspected, excisional biopsy with lidocaine is safe, regardless of gestational status, and therefore should be performed without delay.

- Concerning staging, ultrasonography and magnetic resonance imaging are considered the safest techniques but recent evidence shows that radiographic studies (included computed tomography) can be appropriate, if the benefits clearly outweigh the risks.

- Sentinel lymph node biopsy indication and timing are still debatable: since it has only prognostic significance, it can be acceptable to delay the procedure until after delivery; if performed, technetium $99 \mathrm{~m}$ alone should be used as a marker.

- If surgery requires general anesthesia and cannot be deferred, the second trimester is the safest to perform it, but risk-benefit ratio has to be assessed by a multidisciplinary discussion involving the patient.

- Treatment of metastatic melanoma during pregnancy is difficult, since traditional agents such as dacarbazine and IFN- $\alpha$, as well as target therapy (BRAF and MEK inhibitors) and immunotherapy are contraindicated.

- In regard of fetal development and wellbeing, PAM is not associated to higher risk of preterm or planned birth, cesarean section and stillbirth, being conversely related to increased rates of large for gestational age newborns; placental and fetal metastases are rare.

- A multidisciplinary tailored approach should be always preferred, with referral to highly specialized centers, if possible; thorough counseling of the mother is necessary, to understand her perspectives and priorities.

Melanoma diagnosed during childbearing period or up to 1 year after delivery is defined as pregnancyassociated melanoma (PAM). There is some evidence that PAM has worse prognosis if compared with melanoma in nonpregnant women, although literature is still inconclusive. Many biological mechanisms could explain this behavior, such as hormonal and immune status, increased lymphangiogenesis but also delay in diagnostic and therapeutic management. If PAM is suspected, a prompt excisional biopsy under local anesthesia can be performed regardless of the gestational period. Conversely, additional staging procedures (such as sentinel lymph node biopsy or imaging) and systemic therapy are still debatable during pregnancy. A multidisciplinary tailored approach should be preferred, together with exhaustive counseling of the mother.

First draft submitted: 3 May 2020; Accepted for publication: 15 June 2020; Published online: 31 July 2020

Keywords: diagnosis $\bullet$ immunotherapy $\bullet$ melanoma $\bullet$ pregnancy $\bullet$ pregnancy-associated melanoma $\bullet$ therapy

\section{Background}

Melanoma is one of the most common cancers in young adults, often occurring in women of fertile age [1,2]. Its incidence has been increasing over the last few decades and age-standardized incidence rates in European countries vary from 3.5 to 34.9 per 100,000 women [3]. Moreover it is the most common malignancy in pregnancy, accounting for $31 \%$ of all cancers identified during gestation $[1,4,5]$. Melanomas diagnosed during childbearing or 
up to 1 year after delivery are termed pregnancy-associated melanomas (PAMs), although this definition may vary among different studies [6]. It is debated if PAMs represent entities somewhat different from classic melanomas, with distinct clinical and etiopathological implications, prognosis and management. The aim of this work is to provide an overview of the available evidence on this disputed topic. The literature research included peer-reviewed articles published in English language, in other words, clinical trials or scientific reviews. They were identified by searching electronic databases (MEDLINE and PubMed), using the words 'melanoma', 'pregnancy', 'pregnancy-associated melanoma' till February 2020 and by using reference lists of previously analyzed articles.

\section{Melanocytic lesions during pregnancy}

Increased melanocytic activity and hyperpigmentation can be observed during pregnancy: melasma, linea nigra, genital and areolar darkening are well known [7]. These conditions seem related to increased levels of estrogen, progesterone, beta and alfa melanocyte-stimulating hormone and beta-endorphin [8]. It was therefore hypothesized that benign melanocytic nevi could undergo physiological modifications during gestation, but in accordance to recent findings, there is insufficient evidence to support the presence of clinical or histological changes [9]. Moreover, diameter increase and transient dermoscopic findings, if recorded, are due to skin stretching: indeed they generally regard benign melanocytic nevi located on breast and abdomen [9]. On the contrary, in pregnant patients with dysplastic nevus syndrome, clinical and histologic changes may occur, although data on this aspect are very limited [10]. Summarizing, it is important to underscore that any major modification in melanocytic lesions should not be disregarded as physiological during pregnancy.

Dermoscopy is a noninvasive diagnostic technique that can be performed safely during pregnancy [11-13]. Dermoscopy improves diagnostic accuracy of melanoma [14,15] and allows its recognition at an early stage thanks to the identification of specific dermoscopic criteria [16-18].

An excisional biopsy with histopathological analysis should be promptly performed when a lesion changes its clinical and dermoscopic characteristics in pregnancy [9]. Any unjustified conservative management could lead to diagnostic delay and eventually to worse prognosis.

\section{Prognosis of PAM}

Older literature suggested that PAMs could lead to a poorer prognosis if compared with melanomas in nonpregnant women [19-21]. On the contrary, a recent large-scale study has found no significant difference in the outcomes, including overall survival, disease-free survival and melanoma-specific survival [22] and other research showed similar results [23]. A meta-analysis including studies up to 2013 found a slight but statistically significant increased risk of death in women with PAM (pooled hazard ratio $=1.56,95 \%$ confidence interval: $1.23-1.99$ ) [24]. Only four studies were included in the statistical model and the authors underlined the strong limitations of this evidence, due to eligibility criteria for the articles and lacking data [24,25]. Similarly, a meta-analysis confirmed these results, showing that PAM was associated with a $17 \%$ higher mortality compared with melanoma diagnosed in nonpregnant women (hazard ratio $=1.17,95 \%$ confidence interval: $1.03-1.33, \mathrm{p}=0.02$ ) [26]. In summary, literature is still inconclusive regarding prognosis of PAMs, but there is some evidence of a worse outcome.

\section{Biological aspects of PAM}

Many biological factors could explain a more aggressive behavior of PAM, such as placental growth factor, human chorionic gonadotropin, estrogen, progesterone, relaxin, indolamine 2,3 dioxygenase and many other circulating factors [27,28]. In the initial phase of implant, these substances are involved in inducing immune tolerance toward the fetus, which can be seen as a semiallogenic transplant. Indeed, they seem to favor immune tolerance through a shift to T helper 2 (Th2) cell response [28]. Moreover, this hormonal status facilitates placentation, which implies neoangiogenesis and tissue invasion: all these mechanisms are not surprisingly in common with melanoma and cancer in general [28]. A recent study demonstrated that PAPP-A, normally secreted by placental tissue, could accelerate melanoma growth through the IGF-1 pathway [29].

Interestingly, near parturition, the changes in hormonal and cytokine asset lead to the loss of immune tolerance and promote labor, thanks to prolactin, CRH and visfatin [28]. The function of estrogen is not so clear and seems to play a role either in the induction of immune dormancy (initial phase of pregnancy) and in the break of tolerance (final phase and labor) [28]. All these findings are interesting, although based on in vitro studies and lacking clinical validation. 
In summary, it is possible that, in the first stages of pregnancy, PAMs grow and escape from immunity taking advantage of the favorable hormonal and cytokine status. Another hypothesis that could explain the worse prognosis of PAMs is increased lymphangiogenesis during the gestational period. This circumstance could enhance the metastatic potential of malignancies [30,31], given that tumor lymphangiogensis is a strong independent factor that predicts presence of melanoma metastasis to sentinel lymph nodes [32]. Notably it was hypothesized that prolactin could play a role in the mechanism and this hormone increases in the late part of gestation, thus suggesting to prefer a prompt management of PAMs, avoiding unnecessary delay.

\section{Management of PAM: surgical excision \& staging}

Some clarifications should be made regarding melanoma management during pregnancy. First of all, a multidisciplinary tailored approach should be adopted and different strategies should be chosen depending on tumoral stage and gestational period. It is therefore clear that a correct staging is the essential prerequisite.

Excisional biopsy can be performed without delay and lidocaine is safe for use in pregnancy [33]. The addition of epinephrine is also considered harmless in small amounts and the benefits in terms of patient's comfort seem to outweigh potential risks: despite one dated study suggested an increase in malformations when mothers were exposed to systemic epinephrine during the first trimester, these data were not confirmed by further research [33,34]. It is essential to remember that one of the possible explanations of poorer prognosis of PAM is simply delayed diagnosis and management, therefore procrastination should be avoided [27]. If wide local excision is feasible under local anesthetic, it should not be postponed, because it is a potentially curative procedure [35].

Sentinel lymph node biopsy (SLNB) is considered technically safe after the first trimester using technetium $99 \mathrm{~m}$ and avoiding blue dye, since the latter has anaphylaxis risk $(<1 \%)$ and potential teratogenic effects [27,36]. In fact, radiation exposure for the fetus is minimal (estimated to 5 milligray [mGy]) and doses up to $50 \mathrm{mGy}$ do not increase incidence of malformation $[37,38]$. However, the main issue for SLNB is that general anesthesia is required in most cases, posing the same challenges as lympadenectomy. After a collegial discussion involving the obstetrician, anesthesiologist and neonatologist, assessment of risk-benefit ratio, and thorough patient information, the second trimester is the safest to perform the surgery [35,39]. In the late phase of the third trimester, the procedure should preferably be postponed after delivery, whereas in the early phase of the third trimester the risk of inducing premature labor should be carefully taken into account [35,40]. Fetal heart monitoring is useful and recommended during intraoperative phases and subsequently, in order to accurately monitor fetal wellbeing [40]. It is essential to highlight that SLNB has only prognostic significance and it is not performed with a curative intent. In a European survey regarding 290 physicians ( $80 \%$ dermatologists, $12 \%$ surgeons and $8 \%$ oncologists) almost half of respondents preferred to delay SLNB until after delivery [41]. However, if the patient is entering the last phase of gestation and is potentially eligible for adjuvant treatment, SLNB can be proposed [39].

For completing tumoral staging, ultrasonography, chest radiograph with appropriate shielding and magnetic resonance imaging are safe methods [37]. Gadolinium use should be limited to situations in which the benefits clearly outweigh the possible risks and superparamagnetic iron oxide contrast should not be used [37]. Recent evidence shows that also other radiographic techniques (included computed tomography) and nuclear medicine studies with technetium $99 \mathrm{~m}$ are acceptable, but iodinated contrast should be used only if absolutely necessary to obtain relevant additional diagnostic information [37].

\section{Management of PAM: systemic treatments}

After pathological staging is completed, systemic therapy could be needed in advanced melanoma. Immunotherapy or target therapy (with BRAF and MEK inhibitors) are commonly used in melanoma, but are not indicated during pregnancy and lactation. In a pregnant patient, in adjuvant setting, these drugs should be reasonably avoided or withheld up to delivery [42]. On the other hand, in metastatic PAM, a treatment could be adopted but a multidisciplinary and individualized approach as well as referral to highly specialized centers are essential in these difficult cases [42]. Termination of pregnancy should be discussed with women in the first or second trimester with advanced disease, in order to gain more therapeutic possibilities [39]. The mother's desires obviously play a central role in decision making, but counseling is fundamental and the clinician should provide all the information needed.

Among BRAF inhibitors, dabrafenib is clearly teratogenic; on the other hand, animal studies suggest that vemurafenib can cross the placenta, but without any associated teratogenic effects [43,44]. In regard of MEK inhibition, animal reproduction studies demonstrated that trametinib and cobimetinib can harm the fetus. Therefore combination therapy with BRAF and MEK inhibitors is not an available option for BRAF-mutated PAMs, while 
there are some case reports of pregnant women with metastatic PAM treated with vemurafenib alone, although with scarce results [44-46].

Drugs used in immunotherapy act on PD-1 pathway such as nivolumab and pembrolizumab or on CTLA4 pathway, such as ipilimumab [43]. Their aim is to break immune tolerance for the tumor but they can also hinder maternal tolerance for the fetus, therefore increasing risk of spontaneous abortions. This is proved by animal studies for anti-PD-1 agents that are thus labeled as pregnancy category D for US FDA, but not for anti-CTLA4, labeled as FDA category $\mathrm{C}$. The use of immunotherapy in pregnancy could lead to immune-related adverse effects and cause malformations and endocrine alterations in the newborn such as congenital hypothyroidism [47-49]. However good outcomes without apparent harm to the newborn are also reported for ipilimumab alone [50] and even for ipilimumab-nivolumab combination [48] suggesting that immune-mediated response to the fetus during the therapy could be patient specific.

Last, other therapies such as imatinib, dacarbazine, IFN- $\alpha$, cyclophosphamide and bexarotene are all contraindicated in pregnancy [43].

\section{Fetal wellbeing \& PAM}

In regard of fetal development and wellbeing, a recent study reported that PAM was not associated with increased risk of preterm or planned birth, cesarean section and stillbirth [51]. On the contrary, it was significantly related to increased rates of large for gestational age newborns, thus suggesting possible implication of growth factors that could play a common role for the fetus and the malignancy [51].

Additionally, it should be remembered that metastatic PAM can involve the placenta in exceptional cases and the fetus in even rarer circumstances. Nevertheless, melanoma represents $30 \%$ of placental metastases and $58 \%$ of fetal metastases, being the most common culprit in such cases [35,52]. Careful macroscopic and histopathological examination of fetal adnexa with appropriate immunohistochemical staining is recommended after delivery in women with known or suspected metastatic PAM [38]. In absence of placental involvement, fetal metastasis can be reasonably excluded according to the literature [53]. On the other hand, if there is evidence of placental involvement, thorough examination of the newborn should be performed, including skin inspection, chest radiography, abdominal ultrasound, liver enzymes and lactate dehydrogenase testing, since approximately $22 \%$ of neonates can also show clinical evidence of disease [52]. This latter situation is associated to a very poor prognosis, both maternal and fetal, being an indicator of very high burden of disease [38,52]. In addition, apparently healthy neonates delivered with concomitant placental metastasis represent a high-risk population and follow-up for at least 24 months should be scheduled [52].

\section{Conclusion}

In conclusion, pregnancy-associated melanoma is a vast, debated and complex field. Thorough information and counseling of patient about risks, issues and therapeutic possibilities is fundamental, in order to understand perspectives and priorities of the mother. A multidisciplinary management together with the obstetrician, neonatologist, radiologist, surgeon and anesthesiologist is extremely useful. Because shared and clear guidelines are lacking, each case should be discussed on an individual basis and a tailored approach should be adopted. Further research is needed to understand biological basis of PAM and new potential therapeutic options.

\section{Future perspective}

It is essential to stress that an excisional biopsy can be promptly obtained in pregnant women with suspicious pigmented lesions and this procedure is both staging and therapeutic. With this concept in mind, any unnecessary delay in diagnosis and therapy of PAM will be hopefully minimized in the future and this should improve its prognosis. Regarding management of metastatic disease during gestation, more data are needed to evaluate available treatments: vemurafenib and ipilimumab are the agents less likely to cause harm to the fetus, although they are far from being considered safe. Research on biological mechanisms that foster PAM could shed light on melanoma pathogenesis, leading to new therapeutic opportunities maybe not only related to pregnant patients. 


\section{Financial \& competing interests disclosure}

The authors have no relevant affiliations or financial involvement with any organization or entity with a financial interest in or financial conflict with the subject matter or materials discussed in the manuscript. This includes employment, consultancies, honoraria, stock ownership or options, expert testimony, grants or patents received or pending, or royalties.

No writing assistance was utilized in the production of this manuscript.

\section{Open access}

This work is licensed under the Attribution-NonCommercial-NoDerivatives 4.0 Unported License. To view a copy of this license, visit http://creativecommons.org/licenses/by-nc-nd/4.0/

\section{References}

Papers of special note have been highlighted as: • of interest; $\bullet \bullet$ of considerable interest

1. Sacchetto L, Zanetti R, Comber H et al. Trends in incidence of thick, thin and in situ melanoma in Europe. Eur. J. Cancer 92, 108-118 (2018).

2. Di Meo N, Conforti C, Nan K et al. Primary malignant melanoma of uterine cervix. G. Ital. Dermatol. Venereol. doi:10.23736/S0392-0488.19.06512-X (2020) (Epub ahead of print).

3. Bray F, Colombet M, Mery L et al. Cancer incidence in five continents Vol. XI IARC Scientific Publications (2017).

4. Stensheim H, Møller B, van Dijk T, Fosså SD. Cause-specific survival for women diagnosed with cancer during pregnancy or lactation: a registry-based cohort study. J. Clin. Oncol. 27(1), 45-51 (2009).

5. Andersson TML, Johansson ALV, Fredriksson I, Lambe M. Cancer during pregnancy and the postpartum period: a population-based study. Cancer 121(12), 2072-2077 (2015).

6. Walker JL, Wang AR, Kroumpouzos G, Weinstock MA. Cutaneous tumors in pregnancy. Clin. Dermatol. 34(3), 359-367 (2016).

7. Walker JL, Wang AR, Kroumpouzos G, Weinstock MA. Melanoma in pregnancy. In: Melanoma: A Modern Multidisciplinary Approach. Riker AI (Ed.). 1-652 Springer International Publishing, Cham (2018).

8. Tyler KH. Physiological skin changes during pregnancy. Clin. Obstet. Gynecol. 58(1), 119-124 (2015).

9. Bieber AK, Martires KJ, Driscoll MS, Grant-Kels JM, Pomeranz MK, Stein JA. Nevi and pregnancy. J. Am. Acad. Dermatol. 75(4), 661-666 (2016).

- Provides a review of acceptable changes in melanocitic lesions during pregnancy.

10. Driscoll MS, Martires K, Bieber AK, Pomeranz MK, Grant-Kels JM, Stein JA. Pregnancy and melanoma. J. Am. Acad. Dermatol. 75(4), 669-678 (2016).

11. Conforti C, Giuffrida R, Retrosi C, di Meo N, Zalaudek I. Two controversies confronting dermoscopy or dermatoscopy: nomenclature and results. Clin. Dermatol. 37(5), 597-599 (2019).

- Recent paper discussing the important aspect of nomenclature in dermatoscopic decription.

12. Giuffrida R, Conforti C, Di Meo N, Deinlein T, Guida S, Zalaudek I. Use of noninvasive imaging in the management of skin cancer. Curr. Opin. Oncol. 32(2), 98-105 (2020).

13. Martins-Costa GM, Bakos R. Total body photography and sequential digital dermoscopy in pregnant women. Dermatol. Pract. Concept. 9(2), 126-131 (2019).

14. Argenziano G, Albertini G, Castagnetti F et al. Early diagnosis of melanoma: what is the impact of dermoscopy? Dermatol. Ther. 25(5), 403-409 (2012).

15. Conforti C, Giuffrida R, Vezzoni R, Resende FS, Meo N, Zalaudek I. Dermoscopy and the experienced clinicians. Int. J. Dermatol. 59(1), 16-22 (2020).

16. Resende FS, Conforti C, Giuffrida R, Hamilko de Barros M, Zalaudek I. Raised vulvar lesions: be aware! Dermatol. Pract. Concept. 8(2), 158-161 (2018).

17. Seabra Resende FS, Conforti C, Giuffrida R et al. Balloon cell primary nodular melanoma: dermatoscopy evidences. Dermatol. Pract. Concept. 9(2), 155-156 (2019).

18. Giuffrida R, Uranitsch M, Schmid K, Deinlein T, Favero F, Zalaudek I. Hypomelanotic melanoma detected by the "little red riding hood sign" in a patient with neurofibromatosis type 1. Dermatol. Pract. Concept. 7(4), 71-73 (2017).

19. Pack GT, Scharnagel IM. The prognosis for malignant melanoma in the pregnant woman. Cancer 4(2), 324-334 (1951).

20. Shiu MH, Schottenfeld D, Maclean B, Fortner JG. Adverse effect of pregnancy on melanoma. A reappraisal. Cancer 37(1), 181-187 (1976).

21. Slingluff CL, Reintgen DS, Vollmer RT, Seigler HF. Malignant melanoma arising during pregnancy. Ann. Surg. 211(5), 552-559 (1990).

22. Jones MS, Lee J, Stern SL, Faries MB. Is pregnancy-associated melanoma associated with adverse outcomes? J. Am. Coll. Surg. 225(1), 149-158 (2017). 
23. O’Meara AT, Cress R, Xing G, Danielsen B, Smith LH. Malignant melanoma in pregnancy. Cancer 103(6), 1217-1226 (2005).

24. Byrom L, Olsen C, Knight L, Khosrotehrani K, Green AC. Increased mortality for pregnancy-associated melanoma: systematic review and meta-analysis. J. Eur. Acad. Dermatology Venereol. 29(8), 1457-1466 (2015).

25. Byrom L, Olsen CM, Green AC. Reply: increased mortality for pregnancy-associated melanoma: systematic review and meta-analysis. J. Eur. Acad. Dermatology Venereol. 30(9), 1618-1619 (2016).

26. Kyrgidis A, Lallas A, Moscarella E, Longo C, Alfano R, Argenziano G. Does pregnancy influence melanoma prognosis? A meta-analysis. Melanoma Res. 27(4), 289-299 (2017).

-• A recent meta-analysis which suggests that mortality associated to pregnancy-associated melanoma (PAM) is higher if compared with melanoma in nonpregnant women.

27. Still R, Brennecke S. Melanoma in pregnancy. Obstet. Med. 10(3), 107-112 (2017).

28. Enninga EAL, Holtan SG, Creedon DJ et al. Immunomodulatory effects of sex hormones: requirements for pregnancy and relevance in melanoma. Mayo Clin. Proc. 89(4), 520-535 (2014).

- Provides relevant insight into hormonal and cytokine mechanisms that could foster PAMs during gestation.

29. Prithviraj P, Anaka M, McKeown SJ et al. Pregnancy associated plasma protein-A links pregnancy and melanoma progression by promoting cellular migration and invasion. Oncotarget 6(18), 15953-15965 (2015).

30. Rodero MP, Prignon A, Avril MF, Boitier F, Aractingi S, Khosrotehrani K. Increase lymphangiogenesis in melanoma during pregnancy: correlation with the prolactin signalling pathway. J. Eur. Acad. Dermatology Venereol. 27(1), e144-e145 (2013).

31. Khosrotehrani K, Huu SN, Boitier F et al. Pregnancy promotes melanoma metastasis through enhanced lymphangiogenesis. AJPA 178(4), 1870-1880 (2011).

32. Dadras SS, Lange-Asschenfeldt B, Velasco P et al. Tumor lymphangiogenesis predicts melanoma metastasis to sentinel lymph nodes. Mod. Pathol. 18(9), 1232-1242 (2005).

33. Kouba DJ, Lopiccolo MC, Alam M et al. Guidelines for the use of local anesthesia in office-based dermatologic surgery. J. Am. Acad. Dermatol. 74(6), 1201-1219 (2016).

34. Murase JE, Heller MM, Butler DC. Safety of dermatologic medications in pregnancy and lactation: Part I. Pregnancy. J. Am. Acad. Dermatol. 70(3), 401.e1-401.e14 (2014).

35. Broer N, Buonocore S, Goldberg C et al. A proposal for the timing of management of patients with melanoma presenting during pregnancy. J. Surg. Oncol. 106(1), 36-40 (2012).

36. Cragan JD. Teratogen update: methylene blue? Teratology 60(1), 42-48 (1999).

37. El-sayed Y, Heine RP, Wharton KR. Committee Opinion No. 723. Obstet. Gynecol. 132(3), 786 (2018).

- Recent guidelines focused on eligibility and safety of imaging studies during pregnancy.

38. Schwartz JL, Mozurkewich EL, Johnson TM. Current management of patients with melanoma who are pregnant, want to get pregnant, or do not want to get pregnant. Cancer 97(9), 2130-2133 (2003).

39. Garbe C, Amaral T, Peris K et al. European consensus-based interdisciplinary guideline for melanoma. Part 1: Diagnostics - Update 2019. Eur. J. Cancer 126, 141-158 (2020).

-. These recent European guidelines on melanoma diagnosis provide relevant and shared recommendations about PAM.

40. Andtbacka RHI, Donaldson MR, Bowles TL et al. Sentinel lymph node biopsy for melanoma in pregnant women. Ann. Surg. Oncol. 20(2), 689-696 (2013).

41. Ribero S, Longo C, Dika E et al. Pregnancy and melanoma: a European-wide survey to assess current management and a critical literature overview. J. Eur. Acad. Dermatology Venereol. 31(1), 65-69 (2017).

42. Ziogas DC, Diamantopoulos P, Benopoulou O et al. Prognosis and management of BRAF V600E-mutated pregnancy-associated melanoma. Oncologist. doi:10.1634/theoncologist.2019-0747 (2020) (Epub ahead of print).

43. Grunewald S, Jank A. New systemic agents in dermatology with respect to fertility, pregnancy, and lactation. J. Dtsch Dermatol. Ges. 13(4), 277-290 (2015).

-. Provides information about eligibility and safety of dermatologic therapies during pregnancy.

44. Maleka A, Enblad G, Sjörs G, Lindqvist A, Ullenhag GJ. Treatment of metastatic malignant melanoma with vemurafenib during pregnancy. J. Clin. Oncol. 31(11), e192-e193 (2013).

45. de Haan J, van Thienen JV, Casaer M et al. Severe adverse reaction to vemurafenib in a pregnant woman with metastatic melanoma. Case Rep. Oncol. 11(1), 119-124 (2018).

46. Marcé D, Cornillier H, Denis C, Jonville-Bera A-P, Machet L. Partial response of metastatic melanoma to BRAF-inhibitor-monotherapy in a pregnant patient with no fetal toxicity. Melanoma Res. 29(4), 446-447 (2019).

47. Menzer C, Beedgen B, Rom J et al. Immunotherapy with ipilimumab plus nivolumab in a stage IV melanoma patient during pregnancy. Eur. J. Cancer 104, 239-242 (2018). 
48. Burotto M, Gormaz JG, Samtani S et al. Viable Pregnancy in a patient with metastatic melanoma treated with double checkpoint immunotherapy. Semin. Oncol. 45(3), 164-169 (2018).

49. Xu W, Moor RJ, Walpole ET, Atkinson VG. Pregnancy with successful foetal and maternal outcome in a melanoma patient treated with nivolumab in the first trimester. Melanoma Res. 29(3), 333-337 (2019).

50. Mehta A, Kim KB, Minor DR. Case report of a pregnancy during ipilimumab therapy. J. Glob. Oncol. (4), 1-3 (2018).

51. Bannister-Tyrrell M, Roberts CL, Hasovits C, Nippita T, Ford JB. Incidence and outcomes of pregnancy-associated melanoma in New South Wales 1994-2008. Aust. New Zeal. J. Obstet. Gynaecol. 55(2), 116-122 (2015).

52. Alexander A, Samlowski WE, Grossman D et al. Metastatic melanoma in pregnancy: risk of transplacental metastases in the infant. $J$. Clin. Oncol. 21(11), 2179-2186 (2003).

53. Altman JF, Lowe L, Redman B et al. Placental metastasis of maternal melanoma. J. Am. Acad. Dermatol. 49(6), 1150-1154 (2003). 
